Bio-grafia: Escritos sobre la Biologia y su Enseñanza Vol 3 No5 ISSN 2027-1034. Segundo semestre de 2010, Bogotá, Colombia, pp 83 - 101

\title{
SISTEMA DE CATEGORÍAS PARA ANÁLISIS DIDÁCTICO DE LOS TRABAJOS PRÁCTICOS EN LA ENSEÑANZA DE LA BIOLOGÍA.
}

\section{CATEGORY SYSTEM FOR ANALYSIS OF PRACTICAL TRAINING IN THE TEACHING OF BIOLOGY.}

\begin{tabular}{|l|}
\hline Recibido: $10-08-2010$ \\
\hline Aceptado: 3-12-2010 \\
\hline
\end{tabular}

PUENTES ECHEVERRI MARTHA LUCIA ${ }^{1}$, y VALBUENA USSA ÉDGAR ORLAY $^{2}$

\section{RESUMEN.}

A partir del análisis documental de fuentes especializadas en Didáctica de las Ciencias, se propone un sistema de categorías para la realización de análisis didáctico de los Trabajos Prácticos (TP) en la enseñanza de la Biología. Las categorías propuestas son: naturaleza, finalidad, aspectos metodológicos, evaluación, y relación con aspectos epistemológicos de la Biología y de las Ciencias. Dadas las características del sistema propuesto, éste posibilita la realización de estudios sobre caracterización de TP, estructuración de $\mathrm{TP}$, análisis en la planificación y desarrollo de $\mathrm{TP}$, reflexiones sobre el conocimiento didáctico del contenido biológico en profesores.

PALABRAS CLAVE: Trabajos prácticos, enseñanza de la Biología, análisis didáctico.

\section{CATEGORIES SYSTEM FOR PEDAGOGIC ANALYSIS OF PRACTICAL WORKS IN BIOLOGY TEACHING}

\section{ABSTRACT}

From the documentary analysis of specialized sources in Science education, a category system is proposed for the execution of practical works didactic analysis in Biology teaching. The suggested categories are: nature, aim, methodological aspects, assessment and connection with epistemological aspects of Biology and Sciences. Given the characteristics of the proposed system, this enables the carrying out of studies about the characterization of practical works, their structure, analysis in the

${ }^{1}$ Profesora adscrita a la Secretaría de Educación del Distrito de Bogotá. Integrante del Grupo de Investigación Conocimiento Profesional del Profesor de Ciencias. marthikpuentes@hotmail.com

${ }^{2}$ Profesor Departamento de Biología, Universidad Pedagógica Nacional (Colombia). Coordinador Grupo de Investigación Conocimiento Profesional del Profesor de Ciencias. valbuena@pedagogica.edu.co edgarorlay@hotmail.com 
planning and development of practical works, reflections upon the pedagogical knowledge of the biological content in teachers.

KEYWORDS: practical works, Biology teaching, pedagogical analysis.

\section{INTRODUCCIÓN.}

Una de las actividades que merece especial análisis en la enseñanza de las ciencias y de la Biología en particular, es el Trabajo Práctico (TP) que se realiza en la escuela, dado que éste se puede abordar desde diversas perspectivas y pretensiones (epistemológicas, pedagógicas, didácticas, de gestión de recursos, etc.) y tiene implicaciones respecto a la infraestructura y dotación de laboratorios de las instituciones educativas, la estructura curricular y por supuesto, la práctica y la formación docente. Así, asumimos el TP, como aquellas actividades de enseñanza que implican la interacción de los estudiantes con materiales mediante procedimientos, los cuales conllevan la relación implícita o explícita con los aspectos teóricos, particulares de los contenidos de enseñanza; igualmente, consideramos como TP aquellas vivencias de los estudiantes en el contexto escolar, concretamente las salidas de campo o excursiones, las cuales también implican el establecimiento de relaciones con los referentes teóricos propios de los contenidos de enseñanza. Para efectos del presente artículo abordamos dos tipos de TP: las prácticas de laboratorio y las salidas de campo.

Para el caso concreto de la Biología, es importante tener en cuenta que la manera como se produce el conocimiento en esta ciencia presenta diferencias de orden metodológico en relación con ciencias como la Física y la Química, tal es el caso de la utilización de la narración histórica y las limitaciones en la experimentación. Esto, debido a las especificidades en las características de los fenómenos que estudia dicha ciencia. Consecuentemente, es menester que el abordaje de los TP que se realicen para la enseñanza de la Biología tengan presentes dichas particularidades.

La literatura especializada en Enseñanza de las Ciencias indica que los TP han sido abordados como objeto de investigación de manera rigurosa, contando con visibles grupos de investigadores especialistas en el campo y habiendo producido conocimiento particular al respecto. Así, autores como Porlán (1998) y Gil-Pérez, Carrascosa, y Martínez (2000) consideran que los TP se han constituido en una Línea de Investigación, perteneciente a la Didáctica de las Ciencias. En el mismo sentido, cabe resaltar que la producción respecto a los $\mathrm{TP}$ ha sido tan prolífica que ha conllevado a la realización de publicaciones en libros especializados en el área (Lunneta, 1998; Del Carmen, 2000; Psillos y Niedder, 2002; Caamaño, 2003; Lunneta, Hofstein y Clough, 2007). Para el caso de los TP en la enseñanza de la Biología, una reciente investigación de Valbuena et al (2010) identifica a los TP como una de las problemáticas más abordadas en las publicaciones especializadas en el campo de la enseñanza de esta ciencia.

Es así como se han dedicado diversas investigaciones sobre los $\mathrm{TP}$, analizando y discutiendo su utilidad, pertinencia, diversas formas de abordarse, las visiones de estudiantes y profesores, entre otros. Encontrándose, de una parte puntos a favor de los TP como estrategia de enseñanza (García, Martínez y Mondelo, 1998; Cortel, 1999; Rodrigo et al, 1999; Caamaño,2003; 
entre otros); y en contraste, otros autores que como Hodson (1994) y Barberá y Valdés (1996) quienes hacen una crítica a la incidencia de los TP en el mejoramiento de la enseñanza de las ciencias, proponiendo a la vez alternativas y replanteamientos al respecto. Por ejemplo, Hodson (1994) pone de manifiesto que los docentes no siempre tienen una idea clara acerca de los objetivos que pretenden alcanzar al implementar estos trabajos y que de igual manera no existe un consenso acerca de las razones para que los estudiantes realicen TP.

En este sentido, los TP representan objeto de la práctica profesional de los profesores, siendo deseable que se constituyan objeto de reflexión y de investigación. Esto precisamente es lo que ha motivado el desarrollo de la investigación que presentamos a continuación, la cual consiste en proponer un sistema de categorías que posibilite el análisis didáctico de los TP en la enseñanza de las Ciencias y de la Biología en particular, con miras a la comprensión y mejoramiento de la formulación, desarrollo y evaluación de dichas estrategias metodológicas para la enseñanza. Para tal fin, consideramos aspectos relacionados no sólo con el tipo de trabajos que son realizados, sino los roles que desempeñan cada uno de los participantes, esto a partir de los referentes epistemológicos desde los cuales están planteados, su forma de realización y de evaluación, entre otros aspectos. De tal manera que la realización de este tipo de análisis, pueda llevar a comprender de qué manera se han pensado y estructurado los TP en la escuela, y así poder plantear formas de transformación que conlleven a que estas prácticas favorezcan el aprendizaje significativo no sólo de conceptos básicos en la Biología, sino que permitan a los estudiantes y profesores apropiarse de la naturaleza de la ciencia y en especial de la Biología, alejándolos de visiones deformadas que en muchos casos son transmitidas a través de la realización algorítmica de TP.

\section{ASPECTOS METODOLÓGICOS.}

La formulación del sistema de categorías corresponde a planteamientos de la investigación cualitativa, más concretamente teniendo como referente el análisis documental. En la primera fase de la investigación, a partir de la identificación de publicaciones especializadas en enseñanza de las ciencias, más concretamente referentes a los TP, realizamos un análisis de los aspectos que se abordan en los diferentes estudios. Para tal fin, además de revisar revistas sobre enseñanza de las ciencias (especialmente de España y del ámbito anglosajón), acudimos a la consulta en bases de datos especializadas, empleando los descriptores: science education, practical works, biology teaching, trabajos prácticos, enseñanza de la biología y enseñanza de las Ciencias. Otra fuente de información fundamental revisada estuvo constituida por libros especializados en el área de la Didáctica de las Ciencias.

Posteriormente, en la segunda fase, a partir de la sistematización de los objetos de investigación abordados en las investigaciones y revisiones, diseñamos la propuesta del sistema de categorías, lo cual demandó varias versiones que se fueron replanteando a partir de la discusión al interior del Grupo de Investigación. 


\section{RESULTADOS Y DISCUSIÓN.}

A partir de la revisión bibliográfica, realizamos un análisis encontrando diversas posturas en cuanto a lo que se considera TP, sus características y participantes, el enfoque desde el que se plantea, entre otros. A través de este análisis pudimos identificar categorías comunes en los diferentes planteamientos, y en otros casos algunas categorías que no se contemplaban, o que de cierta manera no eran explícitas, a partir de esto fuimos agrupando la información de acuerdo al área en la que se realizaba el estudio y a la forma como podía aportar al establecimiento de las categorías.

Hay que tener en cuenta que los referentes epistemológicos de la Biología no son abordados de manera clara o explícita en ninguno de los planteamientos revisados, por lo que esta resulta ser una categoría emergente (en comparación con las investigaciones tomadas como referencia) dentro del establecimiento del sistema de categorías. Al respecto, consideramos relevante esta categoría de análisis teniendo en cuenta que puede aportar significativamente a la comprensión y caracterización de este tipo de trabajos dentro de la enseñanza de la Biología, ya que puede llevarnos a comprender en cierta medida sus características y particularidades.

Después de realizar los ajustes y reestructuraciones a las versiones preliminares, finalmente planteamos un sistema compuesto por cinco categorías, las cuales describiremos más adelante. Es necesario aclarar que las categorías y su nominación no corresponden a categorías independientes, sino que podría considerarse que parte de las características de una podrían estar relacionadas con otra(s), incluso en algunos casos contenidas en otra(s). Por ejemplo, la categoría naturaleza de los TP está estrechamente relacionada con la categoría relación con aspectos epistemológicos. La situación a la que estamos haciendo referencia se hace más evidente para el caso de la subcategoría clases de TP, la cual permite interrelacionar las características de la mayoría de las otras categorías y subcategorías (ver Tabla 1). Pese a lo anterior, y con el ánimo de aportar a las investigaciones sobre TP decidimos realizar la caracterización de cada una de las categorías en la idea de contribuir con elementos conceptuales que posibiliten el abordaje de aspectos particulares de los TP.

A continuación presentamos en primera instancia la síntesis del sistema de categorías (ver Figura 1) y posteriormente la caracterización de cada una de las categorías y subcategorías que lo conforman. Además, sintetizamos en la Tabla 1 la relación que existe entre las clases de TP y las diferentes categorías de análisis. 
Bio-grafia: Escritos sobre la Biologia y su Enseñanza Vol 3 No5 ISSN 2027-1034. Segundo semestre de 2010, Bogotá, Colombia, pp 83 - 101

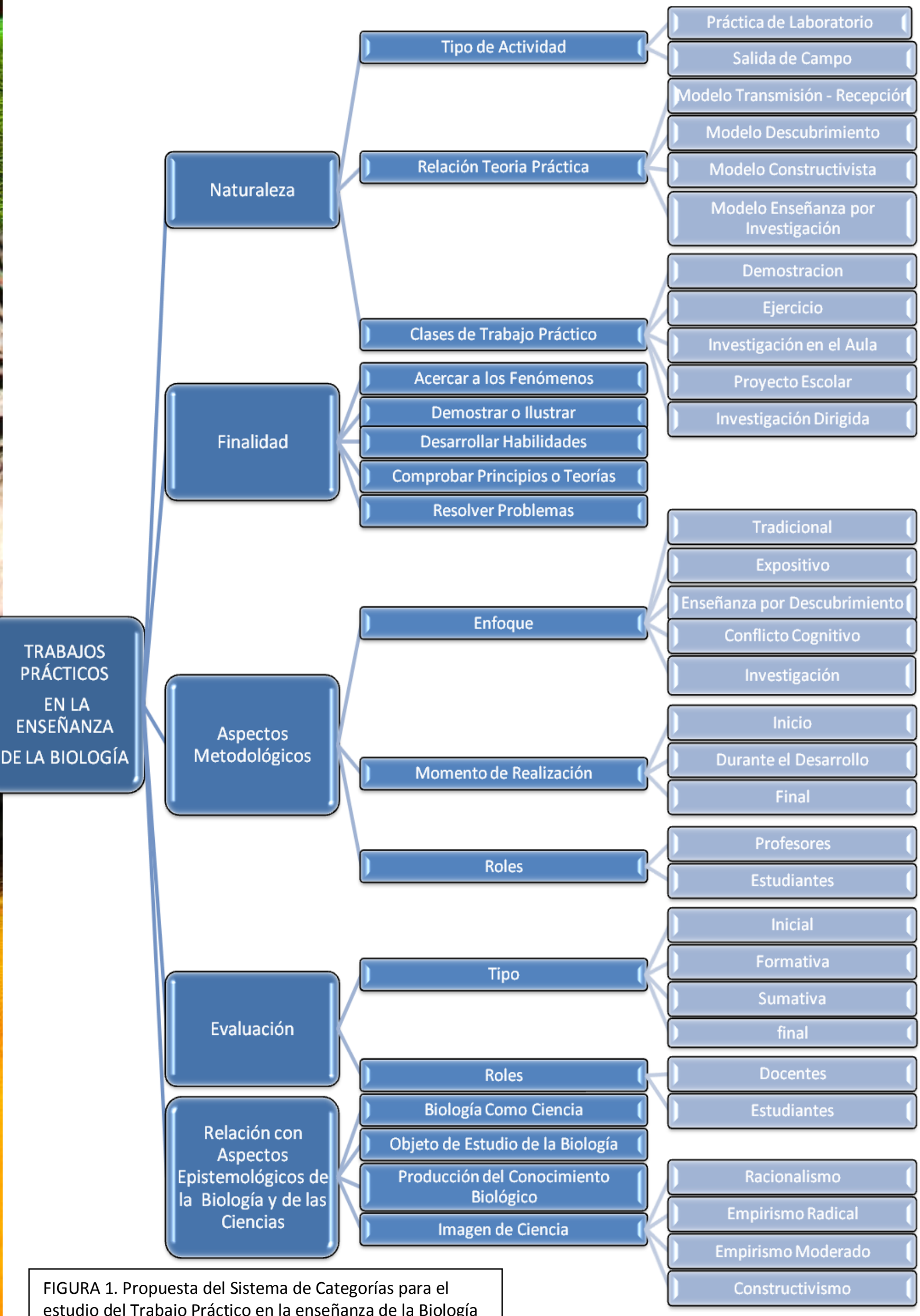
estudio del Trabajo Práctico en la enseñanza de la Biología 
Bio-grafia: Escritos sobre la Biologia y su Enseñanza Vol 3 No5 ISSN 2027-1034. Segundo semestre de 2010, Bogotá, Colombia, pp 83 - 101

\begin{tabular}{|c|c|c|c|c|c|c|}
\hline & & \multicolumn{5}{|c|}{ CLASE DE TRABAJO PRÁCTICO } \\
\hline & & DEMOSTRACIÓN & EJERCICIO & $\begin{array}{l}\text { INVESTIGACIÓN } \\
\text { EN EL AULA }\end{array}$ & $\begin{array}{l}\text { PROYECTO } \\
\text { ESCOLAR }\end{array}$ & $\begin{array}{c}\text { INVESTIGACIÓN } \\
\text { DIRIGIDA }\end{array}$ \\
\hline \multirow{3}{*}{ 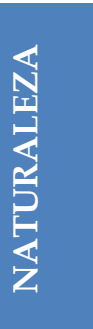 } & $\begin{array}{l}\text { TIPO DE } \\
\text { ACTIVI- } \\
\text { DAD }\end{array}$ & \multicolumn{5}{|c|}{$\begin{array}{l}\text { Trabajo de laboratorio } \\
\text { Salida de campo }\end{array}$} \\
\hline & RELACIÓ & \multirow{2}{*}{$\begin{array}{l}\text { Modelo } \\
\text { transmisión - } \\
\text { recepción }\end{array}$} & \multirow{2}{*}{$\begin{array}{l}\text { Modelo } \\
\text { transmisión - } \\
\text { recepción }\end{array}$} & \multicolumn{3}{|c|}{ Constructivismo } \\
\hline & $\begin{array}{l}\text { TEORIA } \\
\text { PRÁCTIC } \\
\text { A }\end{array}$ & & & $\begin{array}{l}\text { Modelo } \\
\text { enseñanza por } \\
\text { descubrimiento }\end{array}$ & \multicolumn{2}{|c|}{$\begin{array}{l}\text { Modelo enseñanza por } \\
\text { investigación }\end{array}$} \\
\hline 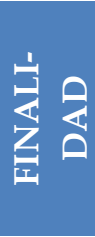 & & $\begin{array}{l}\text { Acercar a los } \\
\text { fenómenos } \\
\text { Demostrar o } \\
\text { ilustrar }\end{array}$ & $\begin{array}{l}\text { Desarrollar } \\
\text { habilidades } \\
\text { Comprobar } \\
\text { principios o } \\
\text { teorías }\end{array}$ & $\begin{array}{l}\text { Resolver } \\
\text { problemas }\end{array}$ & $\begin{array}{l}\text { Resolver } \\
\text { problemas }\end{array}$ & $\begin{array}{l}\text { Plantear y } \\
\text { resolver } \\
\text { problemas }\end{array}$ \\
\hline \multirow[b]{3}{*}{ 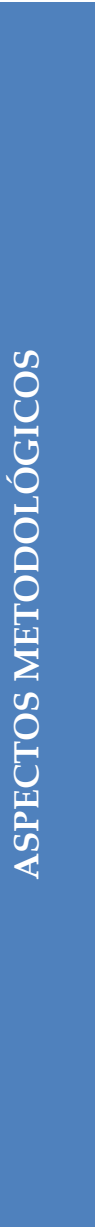 } & ENFOQUE & Tradicional & Expositivo & $\begin{array}{l}\text { Enseñanza por } \\
\text { descubrimiento }\end{array}$ & $\begin{array}{l}\text { Conflicto } \\
\text { cognitivo }\end{array}$ & $\begin{array}{l}\text { Conflicto } \\
\text { cognitivo } \\
\text { Investigación }\end{array}$ \\
\hline & $\begin{array}{l}\text { MOMEN- } \\
\text { TO DE } \\
\text { REALIZA- } \\
\text { CIÓN }\end{array}$ & Finalización & $\begin{array}{l}\text { Inicio } \\
\text { Finalización }\end{array}$ & $\begin{array}{l}\text { Inicio } \\
\text { Durante el } \\
\text { desarrollo } \\
\text { Finalización }\end{array}$ & $\begin{array}{l}\text { Inicio } \\
\text { Durante el } \\
\text { desarrollo } \\
\text { Finalización }\end{array}$ & $\begin{array}{l}\text { Inicio } \\
\text { Durante el } \\
\text { desarrollo } \\
\text { Finalización }\end{array}$ \\
\hline & ROLES & $\begin{array}{l}\text { DOCENTE: } \\
\text { Planea, propone } \\
\text { y realiza } \\
\text { ESTUDIANTE: } \\
\text { observa y toma } \\
\text { nota }\end{array}$ & $\begin{array}{l}\text { DOCENTE: } \\
\text { planea, } \\
\text { propone y } \\
\text { dirige } \\
\text { ESTUDIANT } \\
\text { E: realiza y } \\
\text { sigue } \\
\text { protocolos }\end{array}$ & $\begin{array}{l}\text { DOCENTE: } \\
\text { acompaña y } \\
\text { propone } \\
\text { algunos } \\
\text { problemas } \\
\text { ESTUDIANTE } \\
\text { : descubre } \\
\text { teorías, } \\
\text { propone y } \\
\text { aplica } \\
\text { metodologías } \\
\text { Indaga y busca } \\
\text { respuestas }\end{array}$ & $\begin{array}{l}\text { DOCENTE: } \\
\text { desestabiliza, } \\
\text { plantea } \\
\text { problemas o } \\
\text { metodologías, } \\
\text { acompaña } \\
\text { ESTUDIANTE: } \\
\text { propone } \\
\text { metodología } \\
\text { para solucionar } \\
\text { problemas } \\
\text { Indaga, } \\
\text { comprueba y } \\
\text { busca } \\
\text { respuestas }\end{array}$ & $\begin{array}{l}\text { DOCENTE: } \\
\text { Puede plantear } \\
\text { problemas, } \\
\text { acompaña y } \\
\text { dirige la } \\
\text { investigación, } \\
\text { participa del } \\
\text { proceso } \\
\text { ESTUDIANTE: } \\
\text { plantea y } \\
\text { soluciona } \\
\text { problemas, } \\
\text { propone } \\
\text { metodologías, } \\
\text { construye o } \\
\text { modifica su } \\
\text { conocimiento a } \\
\text { partir de la } \\
\text { experiencia }\end{array}$ \\
\hline $\begin{array}{l}\text { EVA } \\
\text { LUA } \\
\text { CIÓ } \\
\mathbf{N}\end{array}$ & TIPO & Final & Final & \multicolumn{3}{|c|}{ Inicial, formativa, sumativa y final. } \\
\hline$\frac{Z}{0}$ & $\begin{array}{l}\text { BIOLOGÍ } \\
\text { A COMO } \\
\text { CIENCIA }\end{array}$ & \multicolumn{2}{|c|}{$\begin{array}{l}\text { Biología como ciencia } \\
\text { experimental semejante a la } \\
\text { Física y / o Química }\end{array}$} & \multicolumn{3}{|c|}{$\begin{array}{l}\text { Biología como ciencia, con características propias y } \\
\text { con modos de proceder propios }\end{array}$} \\
\hline$\stackrel{4}{\sharp}$ & $\begin{array}{l}\text { OBJETO } \\
\text { DE } \\
\text { ESTUDIO }\end{array}$ & \multicolumn{2}{|c|}{$\begin{array}{l}\text { Con características fijas, y } \\
\text { generalizables }\end{array}$} & \multicolumn{3}{|c|}{$\begin{array}{l}\text { Cambiante, individual y no puede estar sujeto a } \\
\text { generalizaciones radicales. }\end{array}$} \\
\hline
\end{tabular}




\begin{tabular}{l|l|l|l|l|l}
\hline IMAGEN & Racionalismo & Racionalismo & Empirismo & Constructivism & Constructivismo \\
DE & & $\begin{array}{l}\text { Empirismo } \\
\text { CIENCIA }\end{array}$ & modical & $\begin{array}{l}\text { Constructivism } \\
\text { Codo }\end{array}$ & o \\
& & & & \\
& & &
\end{tabular}

Tabla 1: Relación entre las Clases de Trabajo Práctico y las Categorías de Análisis

\section{Categoría 1. Naturaleza del TP en la Enseñanza de la Biología.}

Aunque somos conscientes que al hablar de naturaleza se abarcan una gran cantidad de aspectos, en esta categoría, intentamos dar respuesta a la pregunta ¿Qué tipo de TP se realizan en la enseñanza de la Biología? En ella abordamos aspectos relacionados con el tipo de actividades que son consideradas como TP, la relación establecida por los docentes en cuanto a la teoría y la práctica, y la clase de TP que se realizan en las clases de Biología.

\section{Subcategoría Tipo de Actividad.}

Al hacer referencia al tipo de actividad a desarrollar, en la revisión realizada encontramos que se hace mención a actividades realizadas por los estudiantes bajo la supervisión o guía del docente, donde las actividades permiten establecer relaciones de complementariedad con la teoría, el ambiente cotidiano y el trabajo de las Ciencias, sin ser muy relevante el lugar en donde se realicen dichas prácticas (Barberá y Valdés, 1996). Teniendo en cuenta esto, no solo se consideran TP a las prácticas de laboratorio y las salidas de campo, sino que tal como lo plantea Gandolfi (1992), dentro de esta categoría se incluyen los “experimentos mentales o conceptuales" en los que no hay manipulación de materiales. Sin embargo, nosotros decidimos enmarcar el sistema de categorías tomando como referencias solamente las Salidas de Campo y las Prácticas de Laboratorio, en aras a delimitar nuestro objeto de estudio, ya que consideramos que la enseñanza y el aprendizaje mismo, constituyen una práctica, tanto desde el punto de vista del docente, como del estudiante. Así, optamos por abarcar dentro de la investigación, aquellas actividades que conllevan a la manipulación de materiales y/o el establecimiento de relaciones directas (vivenciales) con el objeto de estudio, ya sea a partir de la experimentación o manipulación directa de los fenómenos, hechos u objetos, ó a través de su observación y análisis. Por lo cual, actividades como talleres, pruebas de lápiz y papel, simulaciones en computador, entre otras, no son tenidas en cuenta en la elaboración de este sistema de categorías, no queriendo decir con esto, que en determinados casos, no pudiesen contemplarse para su análisis.

Como Prácticas de Laboratorio, consideramos aquellas actividades en las cuales los alumnos estudian fenómenos o estructuras biológicas y manipulan materiales, ya sea dentro de un aula o en un laboratorio propiamente dicho. Cabe reiterar que este tipo de actividades no es necesariamente desarrollado en un aula especializada o en un laboratorio totalmente dotado. La principal característica de este TP es que permite el estudio de los fenómenos biológicos (a diferentes niveles: morfología, estructura, funcionamiento, etc.) fuera de sus condiciones naturales, concretamente bajo condiciones de laboratorio (es decir: "controladas"), aunque no necesariamente implique la realización de procesos de experimentación.

Una Salida de Campo, o TP de Campo, lo entendemos como una actividad práctica que se 
realiza en un ambiente diferente al aula de clase, en donde los estudiantes pueden entrar en contacto directo con su objeto de estudio. Generalmente no está acompañado de la experimentación, sino de procesos de observación y descripción. Las actividades que en este tipo de TP se realizan incluyen el establecimiento de relaciones entre organismos in situ, o ex situ, en casos como las visitas a museos, o la realización de excursiones a sitios naturales.

\section{Subcategoría Relaciones Teoría-Práctica en la Enseñanza de la Biología.}

Esta subcategoría toma como referentes de contrastación los planteamientos de Gil (1983), acerca de los tres paradigmas en la enseñanza de las Ciencias, así como las reflexiones de Baldaia (2006) en torno a la relación teoría - práctica.

A este respecto, los autores proponen planteamientos que se pueden enmarcar dentro del modelo de transmisión - recepción, en donde el TP se presenta como complemento de la enseñanza teórica que es transmitida por el docente y que gracias al TP podrá ser no sólo confirmada, sino que servirá, según el modelo, para motivar e interesar a los estudiantes frente al concepto o temática trabajada.

Otros TP están enmarcados en el modelo de aprendizaje por descubrimiento, en donde el objetivo es poner al estudiante en contacto con lo que correspondería a la actividad científica, como manera para descubrir leyes y teorías a través de la experimentación. Se plantean los TP tomando la observación como fuente primordial para producir conocimiento científico.

Por otra parte, existen los TP que corresponden al modelo constructivista, en el cual se concibe el aprendizaje como un proceso dinámico y significativo, teniendo en cuenta los conocimientos y/o las ideas previas de los estudiantes. Desde esta perspectiva, las actividades prácticas se realizan a través de la generación de conflictos, la resolución de problemas o por investigaciones. Desde esta perspectiva las actividades prácticas se realizan tomando como base los referentes teóricos, es decir: la teoría se convierte en la "lente" conceptual que posibilita entender lo práctico. A su vez, lo teórico es alimentado por lo práctico.

Baldaia (2006), considera que existe otro tipo de enfoque, en la perspectiva del modelo de enseñanza por investigación, planteando que el TP debe ser visto desde un enfoque CTS, que contemple lo ecológico y lo ético, que implica no asumir la experimentación como centro del aprendizaje y del conocimiento, sino que se basa en aspectos cotidianos de los estudiantes, y a través de los cuales ellos realizan procesos como prever, observar, explicar, interrogar y argumentar.

Sin el ánimo de encasillar en uno de estos modelos, cualquier TP que se realiza en el contexto de la enseñanza de la Biología, consideramos que constituye un referente útil para la realización de estudios didácticos. Creemos pertinente tener en cuenta que el modelo desde el cual se plantea una actividad práctica depende en gran medida de los objetivos o los fines con 
los cuales se plantea el TP, por tal razón contemplamos que desde la perspectiva de hipótesis de progresión (Porlán y Rivero, 1998), es posible encontrarse con cualquiera de estos referentes ya sea en las prácticas pedagógicas, como en las concepciones de los profesores, y los contenidos de textos escolares. En otras palabras, hemos hecho referencia a características de modelos didácticos, sin embargo consideramos que estos no mantienen relaciones biunívocas excluyentes con el tipo de relación teoría-práctica; podríamos plantear a nivel de hipótesis que cuando se realizan TP en la clase de ciencias, se pueden establecer relaciones con la práctica tales como: la práctica se relaciona con la teoría en tanto la corrobora, mediante la práctica se descubre la teoría, la práctica contribuye a construir teoría. Al respecto, en otras publicaciones de nuestro grupo de investigación ampliamos lo referente a las relaciones teoría-práctica, a partir de la sistematización de datos obtenidos a partir de entrevistas a profesores y de observaciones a prácticas docentes.

Así, nos identificamos con una visión donde las prácticas no corresponden a la simple explicación de la teoría. Consideramos que las relaciones teoría- práctica, deben guardar en el aula de clase proporciones con el trabajo que se realiza en la actividad cotidiana, donde las actividades prácticas se desarrollan como respuesta a preguntas y/o problemas y para poder realizarlas primero es menester proveer de referentes teóricos que den sentido a las actividades prácticas.

En lo que atañe a los factores para hacer posible la visión de relación teoría - práctica con la cual nos identificamos, nos parece fundamental:

La formación epistemológica de los docentes, haciendo referencia al conocimiento de los referentes desde los cuales está construido el conocimiento de la Biología y la didáctica.

$>\quad$ La estructura curricular que facilite las interrelaciones teoría - práctica, tales como no organizar espacios ordinarios curriculares diferentes para abordar un asunto y otro. ${ }^{3}$

> Abordar las temáticas de enseñanza alrededor de problemáticas o ejes problema que permitan la interrelación y aplicación de la teoría y la práctica de manera simultánea y complementaria.

\section{Categoría 2. Finalidades del TP en la Enseñanza de la Biología.}

Esta categoría aborda los objetivos que se pretenden alcanzar con el desarrollo de TP como actividad formativa, teniendo como referente las propuestas de Prietsley (1997), Caamaño (1992) y Herrón (1971), quienes plantean que los objetivos pueden variar, dentro de una gama que depende en gran medida del enfoque que se tenga frente al TP a realizar. Teniendo en cuenta nuestra propuesta de tipos de TP, planteamos cinco finalidades principales, en algunos casos pueden estar implícitas en otras más complejas. Las finalidades que proponemos son:

Acercamiento a los fenómenos: Los TP no están enmarcados dentro de un problema, ni

${ }^{3}$ Tal como ocurrió con algunos planes de estudio en donde la teoría constituía un espacio académico diferente al de la práctica. 
un contexto específico, tan sólo muestran al estudiante un fenómeno como algo terminado y dado casi que como "verdad".

$>$ Demostración o ilustración: Corresponde a actividades prácticas encaminadas a reforzar lo trabajado como componente teórico. Como lo afirma Caamaño (en: Jiménez, 2003: 97), estas actividades están centradas en relacionar variables y determinar propiedades.

Desarrollo de habilidades: TP que se basan en el seguimiento de protocolos, en donde el estudiante manipula de manera hábil técnicas, métodos y materiales sin situarlos dentro de un contexto o problema específico.

Comprobación de principios o teorías: Estos TP acercan al estudiante a la corroboración de la teoría, a partir del seguimiento de métodos específicos, sin que exista un problema a solucionar, ni un contexto claro dentro del cual se realice dicha comprobación.

Resolución de problemas: Estos TP no se centran sólo en la realización de procedimientos experimentales, sino, en la inclusión de aspectos relacionados con la actividad científica. Así, pretenden permitir a los estudiantes tomar decisiones que les permitan transformar y solucionar problemas en un contexto específico (Gil y Valdés, 1996).

Aunque aquí intentamos hacer una diferenciación de los objetivos o finalidades del TP, es importante tener en cuenta que estas finalidades no son excluyentes, sino que pueden ser complementarias, de manera que lleven al estudiante a aproximarse de manera gradual a la forma en que los científicos producen el conocimiento y a reconocer estrategias que le permitan a partir de su conocimiento cotidiano y de sus intereses y/o necesidades proponer y solucionar situaciones problémicas en su entorno, de forma que logre acercarse a la construcción propia o colectiva de un "conocimiento científico escolar".

\section{Categoría 3. Aspectos metodológicos del T.P. en la Enseñanza de la Biología.}

Esta categoría se refiere a la manera como los docentes realizan los TP al enseñar Biología. Contempla aspectos tales como cuándo se hacen, desde qué enfoque son planteados, qué aspectos se tienen en cuenta en la preparación y realización, y los roles que asume cada uno de los participantes en la actividad Práctica de enseñanza-aprendizaje.

\section{Subcategoría Enfoque.}

En esta subcategoría se tiene en cuenta la manera como los docentes abordan los TP que proponen a sus estudiantes, si el desarrollo del TP se da como un proceso algorítmico, o si por el contrario se trata de un proceso heurístico que permite la construcción y retroalimentación del trabajo y de los conceptos biológicos que subyacen al mismo.

A continuación mostramos los diversos enfoques que consideramos base para abordar el TP y a través de los cuales se podría mostrar que un mismo TP puede plantearse como un proceso algorítmico o como un proceso heurístico dependiendo el enfoque que se le dé. 
Desde un enfoque técnico, el TP estará dado por una serie de instrucciones suministras por el docente y que el estudiante debe seguir sin que exista participación de este último en la planeación o desarrollo del trabajo. El docente puede o no plantear una serie de preguntas que van encaminadas a que el estudiante responda puntualmente de acuerdo a la teoría trabajada. Desde este enfoque, los TP serían asumidos durante el desarrollo de una temática o como cierre de esta, ya que al iniciar el docente considera que el estudiante no tiene los conocimientos para realizar dicho trabajo. En concordancia con los planteamientos de Pozo y Gómez (2000), consideramos que este enfoque puede conllevar a la desmotivación de los estudiantes, ya que no toma en cuenta sus intereses.

Los TP podrían ser considerados, de igual manera, desde un enfoque expositivo, desde el cual se plantean, como procesos algorítmicos, a través de los cuales, los estudiantes comprueban o corroboran aquellos datos que han sido expuestos por su profesor. A partir de lo que plantea el docente, los estudiantes desarrollan algunas habilidades en el manejo y/o manipulación de materiales y técnicas de campo o de laboratorio. Una dificultad que plantea este enfoque, es el hecho de considerar que los conocimientos previos del estudiante son erróneos y deben ser cambiados a través del conocimiento impartido por el docente, de manera que no se permite la participación del estudiante en el diseño y preparación, al considerar que sus propuestas pueden ser erróneas, por basarse en sus ideas previas. Por esto, es el docente quien diseña y propone qué metodología a seguir para que los estudiantes se acerquen a lo que para él es correcto.

Desde nuestra experiencia como profesores hemos visto cómo estos enfoques son muy comunes dentro de los TP que se realizan de forma cotidiana en la escuela, y aunque en algunos casos criticamos estos enfoques, es muy común "caer" en ellos.

Otro enfoque desde el cual podemos plantear los TP es el de enseñanza por descubrimiento, según el cual, la mejor manera para que los estudiantes aprendan Ciencia es "haciendo Ciencia". Desde este enfoque los TP llevarían al estudiante a "investigar" y reconstruir los principales acontecimientos científicos, partiendo de sus intereses, aunque en algunos casos es el docente quien genera ese interés y orienta a los estudiantes en ese "descubrimiento". Desde este enfoque los TP deben asumir como metodología en el aula, el enfoque científico de investigación, el cual está basado en situaciones problémicas, en la planeación de metodologías que permitan resolverlas, y en la organización e interpretación de los resultados que se obtienen. Frente a este enfoque se hacen grandes críticas. Como señalan Pozo y Gómez (2000), una de las limitaciones se basa en el hecho de que casi todo aquello que los docentes o estudiantes saben son ideas que han sido descubiertas o construidas por otros y posteriormente comunicadas, de esta manera no habría un verdadero "descubrimiento" a través de los TP en el ámbito escolar. Sin embargo, hay que destacar que desde este enfoque el TP toma una importancia significativa en la construcción del conocimiento escolar, ya que considera que es a partir de la propia experiencia, desde donde el estudiante aprende y construye su conocimiento. 
Si el TP se realiza desde un enfoque de conflicto cognitivo, las ideas previas de los estudiantes adoptan un papel central, ya que es desde sus creencias que se empieza a buscar la manera para dar solución a un problema planteado por el docente o que surge de sus propios intereses o expectativas, así, desde este enfoque, las prácticas serán propuestas, planeadas y desarrolladas por los estudiantes desde su saber cotidiano y enriquecidas y/o modificadas de acuerdo con el conocimiento científico escolar que construye bajo la dirección del docente. El estudiante es capaz de identificar o reconocer las limitaciones de su conocimiento cotidiano, y entrar en "conflicto" con él, de manera que reconoce que es necesario replantear dicho conocimiento y asume nuevas posiciones de acuerdo con un conocimiento científico, llegando a alcanzar un verdadero cambio conceptual.

Otro enfoque desde el que se pueden plantear los TP es el del aprendizaje por investigación, ya sea dirigida o no. Asume que se debe producir no sólo un cambio conceptual, sino también actitudinal y metodológico, y para esto plantea que el aprendizaje de las Ciencias debe procurar el acercamiento del estudiante al trabajo científico. Aquí, el TP cobra gran importancia y trata de situar al estudiante en un contexto similar al de un científico, bajo guía del docente, quien acompaña el proceso y en determinado caso puede tomar parte en su planteamiento, preparación, realización, análisis y socialización. Aunque este enfoque puede tener algunos puntos de encuentro con el enfoque por descubrimiento, no está cargado de las posturas inductivistas y positivistas del descubrimiento. Desde este enfoque, la Ciencia se muestra más como una construcción social, producto del trabajo en equipo y de construcción de teorías y modelos que permitan dar solución a problemas reales dentro de un contexto específico.

\section{Subcategoría Momento de Realización del TP en la Enseñanza de la Biología.}

En esta subcategoría buscamos analizar en qué momento consideran los docentes que es necesaria y oportuna la realización de TP: al inicio, durante el desarrollo de la temática, al finalizarlos, o a lo largo de los tres momentos. Aunque tratamos de hacer una distinción de estos momentos es importante tener en cuenta que de acuerdo con el enfoque desde el cual se planteen los TP, el hecho de que las prácticas se realicen en uno u otro momento adopta un sentido diferente.

Es decir, si nos posicionamos desde un enfoque técnico, podremos pensar que no se realizarán $\mathrm{TP}$ al inicio de una temática ya que se considera que el estudiante debe tener unos conocimientos "científicos" claros antes de realizar dichos trabajos; mientras que si nuestra postura corresponde a un enfoque de descubrimiento, ó de conflicto cognitivo ó de enseñanza por investigación, el hecho de que los TP se realicen al inicio del proceso puede ser tenido en cuenta como una forma de hacer explícitos los conocimientos previos de los estudiantes y sus creencias frente a determinadas situaciones, de manera que sirvan como insumos para el desarrollo de TP posteriores y que guíen y direccionen el camino a seguir. 
Subcategoría Roles de los participantes en el TP en la Enseñanza de la Biología.

Rodrigo, et al (1999) plantean diversos roles de docentes y estudiantes de acuerdo con el enfoque y con el tipo de trabajo que se esté realizando, estableciendo que desde un enfoque tradicional, el profesor actuaría como expositor omnisciente y el papel del estudiante sería de escucha y redescubridor de teorías. Mientras que en enfoques más flexibles, se permitiría una mayor participación de los estudiantes siendo protagonistas del proceso y el docente asumiría el rol de guía y orientador de procesos. En un enfoque tradicional o expositivo, el docente es quien propone y decide qué tipo de TP realizan sus estudiantes y cómo lo hacen, mientras que los estudiantes se limitan a observar y/o a seguir instrucciones, sin que realmente tengan mucha participación en la propuesta o planeación de dicho trabajo. Contrario a lo que ocurriría en los otros enfoques, en los cuales se da una mayor implicación del estudiante, haciéndolo partícipe de la propuesta del TP, desde la formulación del problema a tratar y la búsqueda de metodologías que lleven a su resolución. De igual forma en estos enfoques el docente puede desempeñar varios roles, de manera que facilite la solución de problemas que pueden ser propuestos por él o por sus estudiantes. Desde estos enfoques, el docente es visto más como un guía, un orientador y un compañero de investigación, que colabora y comparte con sus estudiantes en busca de una solución a una problemática dada.

\section{Categoría 4. La evaluación del TP en la Enseñanza de la Biología.}

Con esta categoría se busca analizar las maneras, instrumentos, finalidades, actores y momentos en que se evalúan los TP. Para esto tomamos como referente los planteamientos de Bannet (2000), Geli (1995), García y Martínez (2003) y Campanario (1998). Destacamos los planteamientos hechos por Geli (1995), y retomados por Campanario (1998), quien pone de manifesto tres aspectos importantes a tener en cuenta en la evaluación:

$>\quad$ La búsqueda de una respuesta a un problema o cuestión, aclarando que dependiendo del enfoque esta respuesta puede ser una confirmación de lo que plantea la teoría.

$>\quad$ Los estudiantes realizan las manipulaciones necesarias para resolver la pregunta o problema.

Los alumnos utilizan procesos intelectuales de distintos niveles dependiendo del tipo de trabajo que se proponga y del enfoque desde el cual se haga.

Teniendo en cuenta estos planteamientos, podemos pensar que existen diferentes tipos de evaluación del TP y diversos instrumentos o herramientas que permitan realizar este proceso evaluativo. Sin embargo, es necesario hacer claridad que desde nuestro punto de vista, la evaluación debe constituirse un proceso de continua retroalimentación, en donde profesores y estudiantes construyan en torno a lo obtenido en el desarrollo de las prácticas, no sólo en términos conceptuales, sino procedimentales y actitudinales; es decir que se pueda, a través de la evaluación, dar cuenta del desarrollo de destrezas y/o habilidades de los estudiantes en 
los procesos de predicción, de observación, de síntesis, de comunicación, de trabajo en equipo y de construcción colectiva, entre otros.

\section{Categoría 5. Relación de los TP con los aspectos epistemológicos de la Biología y las Ciencias.}

Pretendemos en esta categoría reconocer las relaciones entre los TP que se realizan en la escuela, y el objeto de estudio de la Biología, la manera en que se produce el conocimiento biológico y la manera como se procede en la actividad científica.

Al hablar de trabajos prácticos en la enseñanza de la Biología, es importante tener en cuenta algunos aspectos, como son, el objeto de estudio de la Biología, el enfoque desde el cual se plantean dichos trabajos, la forma de producción del conocimiento biológico y la imagen de ciencia que se tenga, y se desee construir con los estudiantes.

Si tenemos en cuenta los enfoques desde los que se plantea el TP, podemos afirmar que desde enfoques tradicionales, los trabajos prácticos mantendrán muy arraigada la forma de proceder en ciencias como la Física y la Química, tratando de llevar a los estudiantes a generalizaciones y a la comprobación de leyes o principios, sin considerar las particularidades de los seres vivos y su gran diversidad para responder ante diferentes situaciones. Por otro lado, otros enfoques de carácter más alternativos, darán un manejo más acorde con las particularidades de la Biología, y utilizarán estas mismas para reconocer características de la Ciencia, dando la oportunidad a los estudiantes de comparar diferentes resultados frente a un mismo problema y analizar las causas de estas diferencias, evaluar los posibles "errores" en el desarrollo de metodologías y proponer unas nuevas que conlleven a la solución de los problemas que plantean.

$\mathrm{Al}$ hacer referencia a la producción del conocimiento biológico, retomamos planteamiento de autores como Jiménez (2003). Así, podríamos encontrar cuatro posturas básicas: una que considera que el conocimiento biológico es producto de la observación directa de los fenómenos o de la naturaleza; una segunda, que propone que la experimentación es la base del conocimiento biológico, otro que plantea que es a través del seguimiento de un método científico como se produce conocimiento y una última que plantea que el conocimiento biológico puede ser construido a partir de narraciones históricas.

Si tenemos en cuenta cada una de estas formas de producción de conocimiento podemos afirmar que los TP deben llevar a los estudiantes a entenderlas, promoviendo actividades prácticas enfocadas a mostrar cada uno de estos aspectos. Desde las diferentes clases de TP que hemos propuesto, encontramos actividades prácticas situadas en cada una de estas formas de producir conocimiento biológico. Por ejemplo, si hablamos de ilustraciones se privilegiará la observación como mecanismo de adquisición de conocimiento; mientras que si el TP que se realiza es un ejercicio, se enfatizará en el seguimiento de un método, que es dado por el docente; en las demás clases de trabajo (investigación dirigida, proyecto escolar o investigación en el aula), aunque se tienen en cuenta los procesos anteriores, existirán 
variaciones, como en el caso del planteamiento de los métodos utilizados, los cuales son propuestos por docentes y estudiantes, además incluye como forma de solución de los problemas la experimentación o la narración histórica, dependiendo del problema que se trate.

Consideramos relevante tener presente los aspectos epistemológicos en la planificación y desarrollo de los TP a la hora de enseñar Biología, dado que estos pueden conducir a una imagen deformada del conocimiento biológico, el cual tiene particularidades derivadas en parte de su objeto de estudio como es el carácter único e irrepetible de los seres vivos, así como su carácter cambiante (Canguilhem, 1976), que conlleva a especificidades en la experimentación que le diferencian de ciencias como la Física y la Química, incluso consideramos, hasta el punto de poner en duda si la Biología es una ciencia experimental. En ese mismo sentido, no podemos perder de vista la discusión epistemológica que presentan diferentes autores (Mayr, 1998, 2006; Caponi, 2001; Jiménez, 2003) en relación con las implicaciones derivadas de las particularidades de los fenómenos biológicos que corresponden a las causas remotas y las causas próximas, dependiendo si nos referimos a una Biología Evolutiva, o a una Biología Funcional. Todo ello, cobra importancia en los TP para la enseñanza de la Biología.

Así pues, la Biología es una ciencia, con un cuerpo organizado de conocimientos, que pretende explicar el mundo de lo vivo y los fenómenos que en él se presentan. Los seres vivos, atendiendo a su complejidad no pueden ser estudiados de igual forma que lo inanimado, de lo cual se encargan la Física y la Química. En la naturaleza no existen dos organismos iguales, lo cual dificulta la posibilidad de hablar de un organismo testigo y un organismo modificado como patrones de comparación frente a un experimento, es decir es necesario atender a la individualización de los seres vivos, en donde cada organismo es diferente a otro. Inclusive, como afirma Canguillem (1976), un organismo no es el mismo después de que ha sido manipulado o después de que se le ha extraído alguna de sus partes, ya sea interna o externa. Los seres vivos y sus poblaciones son demasiado complejos para atender a causas únicas, sino que atienden a diversas interacciones que se dan entre ellos, el medio y el tiempo en que se desarrollan.

Los TP en Biología y en su enseñanza han de atender a sus particularidades de esta ciencia, atendiendo dentro de estas particularidades a la especificidad, ya que los seres vivos no pueden ser objeto de generalizaciones y de igual manera sus procesos; en este caso existen variaciones entre los individuos, las variedades, las especies e incluso entre otros animales y el hombre. Todos responden de maneras diferentes ante una determinada condición, contrario a los que ocurre en los fenómenos propios de la Física o la Química. De manera que es menester tener presente que no todo el TP puede conducir a experimentos.

Retomando los anteriores planteamientos, subrayamos que no todos lo fenómenos biológicos pueden ser explicados a través de la experimentación. Por eso, la Biología se sirve de narraciones históricas y de esa misma manera los trabajos prácticos que se plantean en la enseñanza de la Biología, como recurso que permite la aproximación al conocimiento biológico, deben atender a estas particularidades y verse en cierta medida regida por estas. En 
consecuencia, no necesariamente el trabajo práctico debe contemplar la experimentación como forma de acceder al conocimiento biológico, ni a su objeto de estudio, este es el caso de muchas de las prácticas que se hacen en campo y algunas de las que se hacen en el laboratorio, en las cuales no necesariamente se interviene de manera directa a los organismos, sino que parten de otros procesos como la observación directa de los seres vivos para solucionar determinados problemas.

Atendiendo a desarrollos del Grupo de Investigación Conocimiento Profesional del Profesor de Ciencias (Valbuena, 2007; Sánchez, 2007), el objeto de estudio de la Biología puede ser visto desde diversas perspectivas. Desde una visión analítica, en la cual la unidad de estudio está constituida por la suma de partes, y al estudio de estas partes se le da una gran relevancia, asumiendo que para conocer el todo basta con conocer sus partes constituyentes; algunos TP son planteados desde esta visión, principalmente desde enfoques técnicos, en donde se realizan prácticas aisladas tratando de conocer los diferentes sistemas que forman algunos seres vivos. Desde una visión holística, el objeto de estudio de la Biología está representado no por sus partes, sino por el todo, y es el estudio de las relaciones que se dan entre estos constituyentes lo que hace posible el conocer dicho objeto. Y si se considera el objeto de estudio desde una visión sistémica, se tiene en cuenta la emergencia como característica de los sistemas vivientes, destacando el estudio de las interrelaciones, asumiendo además que no todo el conocimiento biológico se produce a partir de la observación, ni de la experimentación, sino que es producto de la construcción conceptual. Desde estas dos últimas visiones (holística y sistémica) podemos encontrar TP planteados desde enfoques constructivas, ya que a partir de problemas se busca entender y atender a los seres vivos en su complejidad.

Para poder analizar los TP que se realizan en la enseñanza de la Biología, es necesario tener claro desde qué visión de Ciencia están planteados, de manera que puedan contribuir a fortalecer en los estudiantes ideas claras de lo que es la Ciencia. Resulta relevante comprender que el referente que tenga el profesor incide en sus prácticas. Dado lo vasto de este asunto y ante las limitaciones de espacio en esta publicación, no profundizamos en la discusión de todo lo que implica la Naturaleza de las Ciencias y su relación con los TP en la enseñanza.

\section{PROYECCIONES Y CONCLUSIONES.}

Si bien es cierto que no podemos hablar de conclusiones de manera estricta, dadas las características de esta investigación, pues como se ha venido expresando a lo largo del escrito, este trabajo se constituye en una propuesta, si es necesario puntualizar en aspectos relacionados con las generalizaciones a las cuales pudimos llegar a través de él y las proyecciones que podemos atribuirle a este Sistema de Categorías. Así, consideramos clave los siguientes aspectos:

Al establecer el sistema de categorías resulta imposible hacer referencia a "categorías puras", ya que cada una de ellas aporta información valiosa para el análisis de otra u otras categorías. 
Las subcategorías clases de trabajo práctico y enfoque, resultan ser integradoras y permiten aclarar en gran medida lo que se pretende con la realización de un determinado Trabajo Práctico.

$>\quad$ La categoría Relación con los aspectos epistemológicos de la Biología y las Ciencias, es emergente (respecto a la revisión realizada en el campo de los TP). Ésta resulta de suma importancia a la hora de la estructuración y desarrollo de TP para la enseñanza de la Biología en el sentido que puede contribuir a comprender que la Biología cuenta con particularidades que la diferencian de otras Ciencias de la Naturaleza.

$>\quad$ Se requiere validar el Sistema de Categorías, mediante la implementación de estudios que utilice dichos sistemas. Al respecto, cabe mencionar que varios trabajos de la Especialización en Enseñanza de la Biología y de la Licenciatura en Biología de la Universidad Pedagógica Nacional en Colombia han recurrido al sistema, resultando de gran utilidad (por ejemplo, Amórtegui y Correa, 2010; Morales, 2010).

$>\quad$ Este sistema de categorías puede aportar al reconocimiento de las características que son deseables dentro de las prácticas en la enseñanza de la Biología, de manera que quienes retomen este estudio y realicen análisis de prácticas en el aula puedan identificar fortalezas y/o dificultades que se presentan y logren replantear dichas prácticas.

$>\quad$ Este sistema de categorías no constituye una "camisa de fuerza" dada la complejidad del proceso de enseñanza y de los TP, pero si puede constituirse en un referente orientador para el estudio y la caracterización de las prácticas que venimos desarrollando en el aula, incluyendo actividades como: diseño de guías de laboratorio y salidas de campo, planificación y desarrollo de visita a museos y jardines botánicos, formulación y desarrollo de proyectos escolares en los que se implementan TP, análisis de TP en textos escolares, análisis de prácticas docentes en las que se implementan TP, etc.

\section{BIBLIOGRAFÍA.}

Amórtegui, E. y Correa, M (2009). Caracterización de la planificación de salidas de campo en un programa de formación inicial de profesores de Biología. Trabajo de Grado. Bogotá: Universidad Pedagógica Nacional.

Baldaia, L. (2006) El Cambio de las Concepciones Didácticas sobre las Prácticas, en la enseñanza de la Biología. Alambique. Didáctica de las Ciencias Experimentales. 47, 23-29.

Banet, E. (2000). La Enseñanza y el Aprendizaje del Conocimiento Biológico En: Perales, F y Cañal, P. (compiladores) Didáctica de las Ciencias Experimentales. Alcoy: Marfil. pp. 449-478.

Barberá, O, y Valdés, P. (1996). El trabajo práctico en la enseñanza de las Ciencias: una revisión. Enseñanza de las Ciencias. 14 (3), 365-379.

Caamaño, A. (1992). Los trabajos prácticos en ciencias experimentales. Aula de innovación educativa. 9, 61-68

Caamaño, A. (2003). Los trabajos prácticos en ciencias. En: Jiménez, M. (Compiladora), Enseñar Ciencias. Barcelona: Graó , p.p. 95-118.

Campanario, J. (1998). Preguntas y respuestas sobre la evaluación de los alumnos en la enseñanza de las ciencias. Enseñanza de las Ciencias. 19, 69-84.

Canguilhem, G. (1976). El conocimiento de la vida. Capítulo "La experimentación en Biología Animal". Barcelona. Anagrama. pp. 15- 43.

Caponi, G. (2001). Biología Funcional vs Biología Evolutiva: ¿Qué tienen ellos que los otros no 
tengan?. Episteme, Poro Alegre, 12, 23-46

Cortel, A. (1999). El trabajo experimental. Cuadernos de pedagogía. 281. 60-63.

Del Carmen, L. (2000). Los trabajos prácticos. En: Perales, F., Cañal, P (Compiladores). Didáctica de las Ciencias Experimentales. Teoría y práctica de la Enseñanza de las Ciencias. Alcoy: Marfil, p.p. 267-288.

Gandolfi, A. (1992). The Conceptual Experiments in the Development of Physics. En: Actas de la International Conference on History of the Physical-Mathematical Sciences and the Teaching of Sciences. Madrid, 9-1 11 Sept. 1922. pp. 124.

García, S y Martínez, C. (2003). Análisis del Trabajo Práctico en Textos Escolares de Primaria y Secundaria. Enseñanza de las Ciencias. Número extra. pp. 5-16.

García, S; Martínez, C y Mondelo, A. (1998) Hacia la Innovación de las Actividades Prácticas desde la Formación del Profesorado. Enseñanza de las Ciencias. 16 (2), 353-366.

Geli, A. (1995). La Evaluación de los Trabajos Prácticos. Alambique. Didáctica de las Ciencias Experimentales. 4 (2), 25-32.

Gil-Pérez, D. (1983). Tres Paradigmas básicos en la enseñanza de las ciencias. Enseñanza de las Ciencias. 1 (1), 26-33.

Gil-Pérez, D.; Carrascosa, J.; y Martínez, F. (2000). La Didáctica de las Ciencias. Una disciplina emergente y un campo específico de investigación. En: Perales, F., Cañal, P. (compiladores). Didáctica de las Ciencias Experimentales. Teoría y práctica de la Enseñanza de las Ciencias. Alcoy: Marfil, p.p. 11-34.

Gil-Pérez, D. y Valdés, P. (1996); La orientación de las prácticas de laboratorio como investigación: Un ejemplo ilustrativo. Enseñanza de las Ciencias, 14 (2), 155-163.

Herrón, M. (1971). The nature of scientific enquiry. School science review. 79, 171-172.

Hodson, D. (1994). Hacia un enfoque más crítico del Trabajo Práctico. Enseñanza de las Ciencias. 12 (3), 299-313.

Jiménez, M. (2003). La enseñanza y el aprendizaje de la Biología. En: Jiménez, M. (Compiladora), Enseñar Ciencias. Barcelona: Graó , p.p. 119-146.

Lunneta, V. (1998). The School Science Laboratory: Historical perspectives and contexts for contemporary teaching. In: Tobin International Handbook of Science Education. Grat Britain: Kluwer Academic Publishers. pp. 249-262.

Lunneta, V; Hofstein, A., y Clough, M. (2007). Learning in the School Science Laboratory: An Analysis of Resarch, Theory and Practice. In: Abell S. y Lederman (Ed.), N. Handbook of Research on Science Education. New York: Lawrence Erlbaum Associates. pp.393-442.

Mayr, E. (1998). Así es la Biología. Barcelona: Debate.

Mayr, E. (2006). Por qué es única la Biología. Consideraciones sobre la autonomía de una disciplina científica. Buenos Aires: Katz.

Morales, D. (2010). Caracterización de visitas escolares a un Museo de Historia Natural. Dos estudios de caso. Trabajo de Grado. Bogotá: Universidad Pedagógica Nacional.

Porlán, R. (1998). Pasado, presente y futuro de la didáctica de las ciencias. Enseñanza de las Ciencias, 16(1), 175-185.

Porlán, R. y Rivero, A. (1998). El conocimiento de los profesores: una propuesta formativa en el área de Ciencias. Sevilla: Díada.

Pozo, J. y Gómez, M. (2000) Aprender y Enseñar Ciencia. Del conocimiento Cotidiano al Conocimiento Científico. Madrid: Morata.

Priestley, W. (1997). The impact of longer term intervention on reforming physical science teachers' approaches to laboratory instruction: seeking a more effective role for laboratory in 
science education. Dissertation Abstracts International. 58 (3), 806.

Psillos, D. y Niedder, H. (2002). Teaching and Learning in the Science Laboratory. Dordrecht, Boston, London: Kluwer Academic Publishers.

Rodrigo, M; Morcillo, J; Borges, R; Calvo, M; Cordero, N; Garcia, F, y Raviolo, A. (1999). Concepciones sobre el Trabajo Práctico de Campo (TPc): Una aproximación al pensamiento de los futuros profesores. Revista Complutense de Educación. 10 (2), 261-285.

Sánchez, P. (2007). Diseño de una Escala Likert para la Indagación de las Concepciones de los Futuros Profesores Sobre El Conocimiento Biológico en el Contexto del Conocimiento Profesional del Profesor de Biología. (Informe de Trabajo de grado). Bogotá: Universidad Pedagógica Nacional.

Valbuena, E. (2007). El Conocimiento Didáctico del Contenido Biológico. Estudio de las concepciones disciplinares y didácticas de futuros docentes de la Universidad Pedagógica Nacional (Colombia). Tesis doctoral. Madrid: Universidad Complutense de Madrid.

Valbuena, E.; Correa, M; Amórtegui, E.; Bernal, S. (2010). Estado del Arte de las Características del Campo de Conocimiento Sobre la Enseñanza de la Biología. Informe de investigación. Bogotá: Centro de Investigaciones, Universidad Pedagógica Nacional. 